\title{
L'Idiot de la famille : repères chronologiques
}

\section{Gilles Philippe}

\section{OpenEdition}

Journals

Édition électronique

URL : http://journals.openedition.org/recherchestravaux/244

DOI : 10.4000/recherchestravaux.244

ISSN : 1969-6434

\section{Éditeur}

UGA Éditions/Université Grenoble Alpes

\section{Édition imprimée}

Date de publication : 15 octobre 2007

Pagination : 177-180

ISBN : 978-2-84310-111-3

ISSN : 0151-1874

\section{Référence électronique}

Gilles Philippe, «L'Idiot de la famille : repères chronologiques », Recherches \& Travaux [En ligne], 71 | 2007, mis en ligne le 15 avril 2009, consulté le 08 septembre 2020. URL : http://

journals.openedition.org/recherchestravaux/244 ; DOI : https://doi.org/10.4000/recherchestravaux 244 


\section{L'Idiot de la famille : repères chronologiques}

Vers I9I5: Sartre, à dix ans, commence à lire Flaubert : «Vingt fois je relus les dernières pages de Madame Bovary ", écrira-t-il dans Les Mots (mais il dira au Magazine littéraire en novembre 1976 : «Ce que j’ai indiqué dans Les Mots n'a aucune importance pour L'Idiot de la famille »). Sartre reprendra cette lecture pendant ses années à l'École normale supérieure (1925-1929).

I938 : Le premier roman de Sartre, La Nausée, multiplie les souvenirs de Flaubert : la ville normande qui sert de cadre, Bouville, est un clin d'œil à Madame Bovary; le nom du protagoniste (Roquentin) semble avoir été trouvé dans L'Éducation sentimentale; le personnage de l'Autodidacte a tout de Bouvard et Pécuchet. Mais surtout le ton et le style de l'œuvre rappellent souvent Flaubert.

I939 : En décembre, Sartre consacre plusieurs pages, fort critiques, à L'Éducation sentimentale dans son 3 e carnet de guerre.

I943 : L'Etre et le Néant annonce une "psychanalyse existentielle» de Flaubert; Sartre relit sa correspondance dans l'édition Charpentier.

1946-1947: Baudelaire et Situations II présentent Flaubert comme l'exempletype de l'écrivain bourgeois incapable de s'engager et responsable devant l'Histoire d'avoir méprisé la classe ouvrière. Un feuillet inédit de Situations II, conservé par la Bibliothèque nationale de France, annonce à nouveau une étude sur Flaubert. «Mais, dans l'ensemble, je n'ai guère pensé à lui de 1943 à I 954 ", déclarera Sartre à la parution de L'Idiot de la famille.

1954-I955: Suite à un pari avec Roger Garaudy, Sartre écrit en trois mois une première étude sur Flaubert : «Garaudy lui avait proposé de confronter sur un point précis l'efficacité des méthodes marxistes et existentialistes; ils avaient choisi d'expliquer chacun à sa manière Flaubert et son œuvre. Sartre écrivit une longue étude fouillée, mais de forme trop négligée pour qu’il 
puisse envisager de la publier», raconte Beauvoir dans La Force des choses. Dans les numéros de mars et d'avril I954, Les Temps modernes publient une étude de J.-B. Pontalis sur «La maladie de Flaubert» qui a retenu l'intérêt de Sartre. La décision de mettre en chantier le Flaubert coïncide avec le premier travail sur Les Mots (les deux projets sont jumeaux et Sartre décrira souvent Flaubert comme son double inversé). Plusieurs passages de cette première étude seront repris, quinze ans plus tard, dans L'Idiot de la famille. L'orientation du texte eût cependant été fort différente ; on comparera par exemple le premier feuillet du manuscrit de I954, qui ne contient qu'une seule allusion à Flaubert («Flaubert est né en I 82 I. Louis XVIII régnait depuis dix ans et la classe des grands propriétaires fonciers s'était en grande partie reconstituée. Pendant la Restauration cette classe devait freiner le développement de la classe ennemie, de la grande bourgeoisie. »), aux lignes qui ouvrent la version publiée ("Quand le petit Gustave Flaubert, égaré, encore "bestial”, émerge du premier âge, les techniques l'attendent. Et les rôles. Le dressage commence : non sans succès, semble-t-il ; personne ne nous dit, par exemple, qu'il ait eu du mal à marcher. Au contraire nous savons que ce futur écrivain a buté quand il s'est agi de l'épreuve primordiale, de l'apprentissage des mots. Nous tenterons de voir, tout à l'heure, s'il eut, dès l'origine, des difficultés à parler. »).

1957 : Questions de méthode parait, en septembre, dans Les Temps modernes (L'Idiot en sera la « suite »). Deux longues séquences y sont consacrées à Flaubert, dont une est particulièrement importante ("Supposons que je veuille étudier Flaubert... »); en 1957 et 1958, la revue annonce la parution d'un article intitulé « Gustave Flaubert », puis «L'idiot de la famille »; le texte ne paraitra jamais.

1960: Dans une interview à Madeleine Chapsal, après la parution de Critique de la Raison dialectique, Sartre explique qu'il avait besoin de penser la dialectique pour ensuite écrire sur Flaubert.

Fin 1961 : Sartre reprend son travail sur Flaubert; dans les interviews qu'il est amené à donner, il parle de plus en plus de son projet sur Flaubert. À l'exception des quelques mois consacrés à la récriture des Mots, Sartre travaillera désormais presque exclusivement à L'Idiot de la famille, dont il rédigera plusieurs versions successives.

1964-1965: Le succès des Mots (1963) est considérable et Sartre obtient le prix Nobel de littérature (1964). Il rédige une nouvelle version de l'étude sur Flaubert.

I966: Deux articles, sans doute les extraits les plus aboutis de la seconde version de l'ouvrage, et donnés comme des extraits d'un Flaubert à venir paraissent dans les Temps modernes («La conscience de classe chez Flaubert», $\mathrm{n}^{\circ} 240$, mai, p. I92 I-I 952 , et n ${ }^{\circ} 24$ I, juin, p. 2 I I3-2I 53 ; «Flaubert: du poète 
à l'Artiste », $\mathrm{n}^{\circ}$ 243, août, p. I 97-243, $\mathrm{n}^{\circ}$ 244, septembre, p. 423-48 I, et $\mathrm{n}^{\circ} 245$, octobre, p. 598-564). Le contenu de ces textes, largement révisé, sera principalement intégré au tome III de L'Idiot de la famille.

I968: Vers la fin de l'année, Sartre décide de récrire entièrement son travail sur Flaubert. Malgré la désapprobation de son entourage mao, deux années y seront presque entièrement consacrées. Pour écrire, Sartre abuse de Corydrane, d'alcool et de tabac ; Beauvoir relit.

I97I : Les deux premiers tomes de L'Idiot de la famille, divisés en trois parties, paraissent le I 2 mai dans la «Bibliothèque de philosophie » des éditions Gallimard. Le I4, Sartre donne une longue interview à Michel Contat et Michel Rybalka pour le Monde: «J'ai voulu montrer un homme et montrer une méthode » (le texte sera repris en 1976 dans Situations $X$ sous le titre "Sur L'Idiot de la famille »). Il parle du plaisir qu'il a à écrire ce livre et se déclare « sûr de pouvoir terminer le Flaubert». La presse salue la parution de l'ouvrage (le livre fait la une du TLS le 24 septembre), mais surtout comme un objet de curiosité ; les nombreux comptes rendus ne perçoivent pas la dimension intellectuelle du projet : proposer la reconstitution par empathie d'une expérience névrotique. Pendant l'été, à Rome, Sartre achève le tome III.

1972 : Le tome III de L'Idiot de la famille paraît le 29 juin. En élargissant la perspective à de nombreux écrivains du Second Empire, il rompt par de nombreux aspects avec les deux tomes précédents : la névrose de Flaubert est ici envisagée dans son cadre historique et social. Ce tome ne reprend d'ailleurs pas le système de divisions en « parties » qui structurait les deux premiers, mais se décompose en deux livres. Pendant l'année, Sartre travaille au tome IV, qui doit être une étude textuelle de Madame Bovary. Ce complément lui paraît indispensable, mais ne l'intéresse guère ; ses amis maos et surtout son secrétaire Pierre Victor (Benny Lévy) cherchent à le dissuader de prolonger un travail dont ils ne voient guère la pertinence dans la situation historique.

I973 : Sartre abandonne la rédaction du tome IV sans doute au début de l'année ; il devient presque aveugle en juin, mais recommence à prendre des notes pour la fin de L'Idiot, sans pouvoir se relire.

I974 : Pendant l'été, Sartre renonce définitivement au tome IV de L'Idiot; « ce Flaubert inachevé me pèse comme un remords », mais «l'essentiel » est dit, déclarera-t-il au Nouvel Observateur en juin i 975.

I976 : Le 6 mai, Sartre accorde à Michel Sicard un important entretien sur L'Idiot de la famille; le texte paraîtra, avec des notes inédites du tome IV, dans le Magazine littéraire de novembre (il sera repris dans M. Sicard, Essais sur Sartre, Galilée, I 989).

I977 : La première traduction de l'ouvrage paraît en Allemagne (vingt ans plus tard en anglais ; toujours pas de traduction complète en italien et dans les 
autres langues importantes, bien que des contrats aient rapidement été signés à la parution).

I980: Sartre meurt le is avril.

I982 : La fille adoptive de Sartre, Arlette Elkaïm-Sartre, fait don à la Bibliothèque nationale de France d'un lot de 900 feuillets manuscrits, correspondant à la préface de L'Idiot et aux livres 2 et 3 de la ze partie.

I985 : La BNF acquiert l'essentiel des manuscrits donnés par Sartre à Michèle Vian. Parmi ceux-ci figure un lot d'environ 5000 ff. d'avant-textes de L'Idiot, correspondant à la ire partie et au ier livre de la ze partie de L'Idiot.

I988 : Arlette Elkaïm-Sartre fait paraître une nouvelle édition revue et complétée de L'Idiot de la famille (la i re édition se serait vendue à un peu moins de 30000 exemplaires, en chiffres cumulés des trois tomes) ; le tome III présente désormais, en annexe, les cahiers que Sartre a tenus en 1972 en vue de l'étude de Madame Bovary qui devait former le tome IV de l'ouvrage. Cette édition ne reproduit cependant pas les notes strictement documentaires prises par Sartre : listes de références et de citations, notamment puisées dans l'édition Conard de la correspondance de Flaubert.

I989: La dation de la fille adoptive de Simone de Beauvoir, Sylvie Le Bonde Beauvoir, fait entrer à la BNF, un lot de I 900 feuillets manuscrits, correspondant à la ze partie de L'Idiot (tome II) et aux deux livres qui forment le tome III.

2005 : À la clôture de l'exposition organisée par la BNF pour le centenaire de Sartre, Arlette Elkaïm-Sartre fait don à l'institution du premier des deux cahiers des notes sur Madame Bovary appelés à devenir le quatrième tome de L'Idiot de la famille. 